\title{
MÜNTZ-LEGENDRE POLYNOMIAL SOLUTIONS OF LINEAR DELAY FREDHOLM INTEGRO-DIFFERENTIAL EQUATIONS AND RESIDUAL CORRECTION
}

\author{
Şuayip Yüzbaş1 ${ }^{1}$, Emrah Gök ${ }^{2, *}$ and Mehmet Sezer $^{3}$ \\ ${ }^{1}$ Department of Mathematics, Faculty of Science, Akdeniz University, 07058, \\ Antalya, Turkey \\ ${ }^{2}$ Department of Mathematics, Faculty of Science, Muğla Sitkı Koçman University, \\ 48000, Muğla, Turkey \\ ${ }^{3}$ Department of Mathematics, Faculty of Science, Celal Bayar University, 45000, \\ Manisa, Turkey \\ syuzbasi@akdeniz.edu.tr, mathman48@hotmail.com, mehmet.sezer@bayar.edu.tr
}

\begin{abstract}
In this paper, we consider the Müntz-Legendre polynomial solutions of the linear delay Fredholm integro-differential equations and residual correction. Firstly, the linear delay Fredholm integro-differential equations are transformed into a system of linear algebraic equations by using by the matrix operations of the Müntz-Legendre polynomials and the collocation points. When this system is solved, the MüntzLegendre polynomial solution is obtained. Then, an error estimation is presented by means of the residual function and the Müntz-Legendre polynomial solutions are improved by the residual correction method. The technique is illustrated by studying the problem for an example. The obtained results show that error estimation and the residual correction method is very effective.
\end{abstract}

Key Words- Linear delay Fredholm integro-differential equation, Müntz-Legendre polynomials, collocation method, residual function.

\section{INTRODUCTION}

In this study, for the linear delay Fredholm integro-differential equations $[1,7]$

$$
\sum_{k=0}^{m} P_{k}(x) y^{(k)}\left(x+\tau_{k}\right)=g(x)+\int_{0}^{1} \sum_{s=0}^{m} K_{s}(x, t) y^{(s)}\left(x+\gamma_{s}\right) d t, \quad 0 \leq x, t \leq 1
$$

under the boundary conditions

$$
\sum_{k=0}^{m-1}\left(a_{j k} y^{(k)}(0)+b_{j k} y^{(k)}(1)\right)=\lambda_{j}, j=0,1, \ldots, m-1,
$$

the approximate solution based on the Müntz-Legendre polynomials will be obtained in the form

$$
y_{N}(x)=\sum_{n=0}^{N} a_{n} L_{n}(x) .
$$

Here, $y^{(0)}(x)=y(x)$ is the unknown function, $P_{k}(x)$ and $g(x)$ are the functions defined on interval $0 \leq x \leq 1$ and $a_{j k}, b_{j k}, \lambda_{j} \tau_{k}, \gamma_{s}$ are real constants, $a_{n}$ $(n=0,1,2, \ldots, N)$ is the unknown Müntz-Legendre coefficients; $N$ is any positive 
integer and $L_{n}(x),(n=0,1,2, \ldots)$ denote the Müntz-Legendre polynomials [6] defined by

$$
L_{n}(x)=\sum_{j=n}^{N}(-1)^{N-j}\left(\begin{array}{l}
N+1+j \\
N-n
\end{array}\right)\left(\begin{array}{l}
N-n \\
N-j
\end{array}\right) x^{j}, \quad K \leq n \leq N, 0 \leq x \leq 1 .
$$

Also, an error problem is constructed by the residual error function and the MüntzLegendre polynomials of this problem are computed and thus the error function is estimated by these solutions. And then, the approximate solutions are improved by summing the Müntz-Legendre polynomial solutions and the estimated error function [4].

\section{FUNDAMENTAL MATRIX RELATIONS}

Let us consider the equation (1) and find the matrix forms of each term in the equation. For this purpose let us write the matrix form of the differential part on the left hand side of the equation. First we can write the approximate solution (3) in the matrix form [5] as,

$$
y(x)=\mathbf{L}(x) \mathbf{A}
$$

where

$$
\mathbf{L}(x)=\left[\begin{array}{llll}
L_{0}(x) & L_{1}(x) & \ldots & L_{N}(x)
\end{array}\right] \text { and } \mathbf{A}=\left[\begin{array}{llll}
a_{0} & a_{1} & \ldots & a_{N}
\end{array}\right]^{T} .
$$

Here, the matrix $\mathbf{L}(x)$ can be written as

$$
\mathbf{L}(x)=\mathbf{X}(x) \mathbf{F}^{T}
$$

so that $\mathbf{X}(x)=\left[\begin{array}{llll}1 & x & \ldots & x^{N}\end{array}\right]$ and

$$
\mathbf{F}=\left[\begin{array}{cccccc}
(-1)^{N}\left(\begin{array}{c}
N+1 \\
N
\end{array}\right) & (-1)^{N-1}\left(\begin{array}{c}
N+2 \\
N
\end{array}\right)\left(\begin{array}{c}
N \\
N-1
\end{array}\right) & (-1)^{N-2}\left(\begin{array}{c}
N+3 \\
N
\end{array}\right)\left(\begin{array}{c}
N \\
N-2
\end{array}\right) & \cdots & (-1)^{1}\left(\begin{array}{c}
2 N \\
N
\end{array}\right)\left(\begin{array}{c}
N \\
1
\end{array}\right) & (-1)^{0}\left(\begin{array}{c}
2 N+1 \\
N
\end{array}\right) \\
0 & (-1)^{N-1}\left(\begin{array}{c}
N+2 \\
N-1
\end{array}\right) & (-1)^{N-2}\left(\begin{array}{c}
N+3 \\
N-1
\end{array}\right)\left(\begin{array}{c}
N-1 \\
N-2
\end{array}\right) & \cdots & (-1)^{1}\left(\begin{array}{c}
2 N \\
N-1
\end{array}\right)\left(\begin{array}{c}
N-1 \\
1
\end{array}\right) & (-1)^{0}\left(\begin{array}{c}
2 N+1 \\
N-1
\end{array}\right) \\
0 & 0 & (-1)^{N-2}\left(\begin{array}{c}
N+3 \\
N-2
\end{array}\right) & \cdots & (-1)^{1}\left(\begin{array}{c}
2 N \\
N-2
\end{array}\right)\left(\begin{array}{c}
N-2 \\
1
\end{array}\right) & (-1)^{0}\left(\begin{array}{c}
2 N+1 \\
N-2
\end{array}\right) \\
0 & 0 & 0 & \ddots & \vdots & \vdots \\
\vdots & \vdots & \vdots & \cdots & (-1)^{1}\left(\begin{array}{c}
2 N \\
1
\end{array}\right) & (-1)^{0}\left(\begin{array}{c}
2 N+1 \\
1
\end{array}\right) \\
0 & 0 & 0 & \cdots & 0 & (-1)^{0}\left(\begin{array}{c}
2 N+1 \\
0
\end{array}\right)
\end{array}\right]
$$

By putting Eq.(6) into Eq.(5), we have the matrix form

$$
y(x)=\mathbf{X}(x) \mathbf{F}^{T} \mathbf{A} .
$$

The $k$ th-order derivative of Eq.(7) is given by

$$
y^{(k)}(x)=\mathbf{X}(x) \mathbf{B}^{k} \mathbf{F}^{T} \mathbf{A}
$$

where 


$$
\mathbf{B}=\left[\begin{array}{ccccc}
0 & 1 & 0 & \cdots & 0 \\
0 & 0 & 2 & \cdots & 0 \\
\vdots & \vdots & \vdots & \ddots & \vdots \\
0 & 0 & 0 & \cdots & N \\
0 & 0 & 0 & \cdots & 0
\end{array}\right] .
$$

By placing $x \rightarrow x+\beta_{j}$ in Eq. (8), we obtain the matrix form

$$
y^{(k)}\left(x+\beta_{j}\right)=\mathbf{X}(x) \mathbf{B}\left(\beta_{j}\right) \mathbf{B}^{k} \mathbf{F}^{T} \mathbf{A}
$$

where

$$
\mathbf{X}\left(x+\beta_{j}\right)=\mathbf{X}(x) \mathbf{B}\left(\beta_{j}\right) \text { and }
$$
for $\beta_{j} \neq 0$ :

and for $\beta_{j}=0$ :

$$
\mathbf{B}\left(\beta_{j}\right)=\left[\begin{array}{ccccc}
\left(\begin{array}{l}
0 \\
0
\end{array}\right)\left(\beta_{j}\right)^{0} & \left(\begin{array}{l}
1 \\
0
\end{array}\right)\left(\beta_{j}\right)^{1} & \left(\begin{array}{l}
2 \\
0
\end{array}\right)\left(\beta_{j}\right)^{2} & \ldots & \left(\begin{array}{l}
N \\
0
\end{array}\right)\left(\beta_{j}\right)^{N} \\
0 & \left(\begin{array}{l}
1 \\
1
\end{array}\right)\left(\beta_{j}\right)^{0} & \left(\begin{array}{l}
2 \\
1
\end{array}\right)\left(\beta_{j}\right)^{1} & \ldots & \left(\begin{array}{l}
N \\
1
\end{array}\right)\left(\beta_{j}\right)^{N-1} \\
0 & 0 & \left(\begin{array}{l}
2 \\
2
\end{array}\right)\left(\beta_{j}\right)^{0} & \ldots & \left(\begin{array}{c}
N \\
2
\end{array}\right)\left(\beta_{j}\right)^{N-2} \\
\vdots & \vdots & \vdots & \ldots & \vdots \\
0 & 0 & 0 & \ldots & \left(\begin{array}{c}
N \\
N
\end{array}\right)\left(\beta_{j}\right)^{0}
\end{array}\right]
$$

$$
\mathbf{B}(0)=\left[\begin{array}{cccc}
1 & 0 & \cdots & 0 \\
0 & 1 & \cdots & 0 \\
\vdots & \vdots & \ddots & \vdots \\
0 & 0 & \cdots & 1
\end{array}\right]_{(N+1) \times(N+1)} .
$$

Now let us construct the matrix form of the integral part on the right hand side of the equation. The kernel function $K_{s}(x, t)$ can be approximated by the truncated Taylor series [2] and the truncated Müntz-Legendre series

$$
K_{s}(x, t)=\sum_{m=0}^{N} \sum_{n=0}^{N}{ }^{T} k_{m n}^{s} x^{m} t^{n} \text { and } K_{s}(x, t)=\sum_{m=0}^{N} \sum_{n=0}^{N}{ }^{L} k_{m n}^{s} L_{m}(x) L_{n}(t)
$$

where

$$
{ }^{T} k_{m n}^{s}=\frac{1}{m ! n !} \frac{\partial^{m+n} K(0,0)}{\partial x^{m} \partial t^{n}}, m, n=0,1,2, \ldots, N, \quad s=0,1,2, \ldots, m
$$

We write the expressions in (10) in the form

$$
K_{s}(x, t)=\mathbf{X}(x) \mathbf{K}_{T}^{s} \mathbf{X}^{T}(t), \quad \mathbf{K}_{T}^{s}=\left[{ }^{T} k_{m n}^{s}\right]
$$

and

$$
K_{s}(x, t)=\mathbf{L}(x) \mathbf{K}_{L}^{s} \mathbf{L}^{T}(t), \quad \mathbf{K}_{L}^{s}=\left[{ }^{L} k_{m n}^{s}\right] .
$$

From equations (6), (11) and (12),

$$
\begin{gathered}
\mathbf{X}(x) \mathbf{K}_{T}^{s} \mathbf{X}^{T}(t)=\mathbf{L}(x) \mathbf{K}_{L}^{s} \mathbf{L}^{T}(t) \Rightarrow \mathbf{X}(x) \mathbf{K}_{T}^{s} \mathbf{X}^{T}(t)=\mathbf{X}(x) \mathbf{F}^{T} \mathbf{K}_{L}^{s} \mathbf{F} \mathbf{X}^{T}(t) \\
\mathbf{K}_{T}^{s}=\mathbf{F}^{T} \mathbf{K}_{L}^{s} \mathbf{F} \text { or } \mathbf{K}_{L}^{s}=\left(\mathbf{F}^{T}\right)^{-1} \mathbf{K}_{T}^{s} \mathbf{F}^{-1}
\end{gathered}
$$


By writing the matrix forms (9) and (13) into the integral part in the equation, we have the matrix relation we have

$$
\begin{aligned}
\int_{0}^{1} \sum_{s=0}^{m} K_{s}(x, t) y^{(s)}\left(x+\gamma_{s}\right) d t & =\int_{0}^{1} \sum_{s=0}^{m} \mathbf{L}(x) \mathbf{K}_{L}^{s} \mathbf{L}^{T}(t) \mathbf{X}(t) \mathbf{B}\left(\gamma_{s}\right) \mathbf{B}^{s} \mathbf{F}^{T} \mathbf{A} d t \\
& =\sum_{s=0}^{m} \int_{0}^{1} \mathbf{L}(x) \mathbf{K}_{L}^{s} \mathbf{L}^{T}(t) \mathbf{X}(t) \mathbf{B}\left(\gamma_{s}\right) \mathbf{B}^{s} \mathbf{F}^{T} \mathbf{A} d t \\
& =\sum_{s=0}^{m} \mathbf{L}(x) \mathbf{K}_{L}^{s} \mathbf{Q}_{s} \mathbf{A}
\end{aligned}
$$

where

$$
\begin{gathered}
\mathbf{Q}_{s}=\int_{0}^{1} \mathbf{L}^{T}(t) \mathbf{X}(t) \mathbf{B}\left(\gamma_{s}\right) \mathbf{B}^{s} \mathbf{F}^{T} d t \\
=\int_{0}^{1} \mathbf{F} \mathbf{X}^{T}(\mathbf{t}) \mathbf{X}(t) \mathbf{B}\left(\gamma_{s}\right) \mathbf{B}^{s} \mathbf{F}^{T} d t \\
=\mathbf{F} \mathbf{H B}\left(\gamma_{s}\right) \mathbf{B}^{s} \mathbf{F}^{T} ; \\
\mathbf{H}=\int_{0}^{1} \mathbf{X}^{T}(t) \mathbf{X}(t) d t=\left[h_{r s}\right] ; h_{r s}=\frac{1}{r+s+1}, r, s=0,1,2, \ldots, N .
\end{gathered}
$$

We put the matrix form (6) into the equation (14) we have the matrix relation,

$$
\int_{0}^{1} \sum_{s=0}^{m} K_{s}(x, t) y^{(s)}\left(x+\gamma_{s}\right) d t=\sum_{s=0}^{m} \mathbf{X}(x) \mathbf{F}^{T} \mathbf{K}_{L}^{s} \mathbf{Q}_{s} \mathbf{A} .
$$

Finally we can obtain the matrix relations for conditions by means of the relation (8)

$$
\sum_{k=0}^{m-1}\left(a_{j k} \mathbf{X}(0)+b_{j k} \mathbf{X}(1)\right) \mathbf{B}^{k} \mathbf{F}^{T} \mathbf{A}=\left[\lambda_{j}\right], j=0,1, \ldots, m-1 .
$$

\section{METHOD OF SOLUTION}

We are now ready to construct the fundamental matrix equation $[8,9]$ for the Eq.(1). For this purpose we substitute the relations (9) and (14) into the Eq.(1) and thus we obtain the matrix equation

$$
\sum_{k=0}^{m} P_{k}(x) \mathbf{X}(x) \mathbf{B}\left(\tau_{k}\right) \mathbf{B}^{k} \mathbf{F}^{T} \mathbf{A}=g(x)+\sum_{s=0}^{m} \mathbf{L}(x) \mathbf{K}_{L}^{s} \mathbf{Q}_{s} \mathbf{A} .
$$

The collocation points defined by

$$
x_{i}=\frac{1}{N} i, \quad i=0,1, \ldots, N
$$

are substituted into Eq. (15) and thus we obtain the system

$$
\sum_{k=0}^{m} P_{k}\left(x_{i}\right) \mathbf{X}\left(x_{i}\right) \mathbf{B}\left(\tau_{k}\right) \mathbf{B}^{k} \mathbf{F}^{T} \mathbf{A}=g\left(x_{i}\right)+\sum_{s=0}^{m} \mathbf{L}\left(x_{i}\right) \mathbf{K}_{L}^{s} \mathbf{Q}_{s} \mathbf{A}
$$

or briefly the fundamental matrix equation becomes

$$
\left\{\sum_{k=0}^{m} \mathbf{P}_{k} \mathbf{X B}\left(\tau_{k}\right) \mathbf{B}^{k} \mathbf{F}^{T}-\sum_{s=0}^{m} \mathbf{X F}^{T} \mathbf{K}_{L}^{s} \mathbf{Q}_{s}\right\} \mathbf{A}=\mathbf{G}
$$


where

$$
\mathbf{P}_{k}=\left[\begin{array}{cccc}
P_{k}\left(x_{0}\right) & 0 & \cdots & 0 \\
0 & P_{k}\left(x_{1}\right) & \cdots & 0 \\
\vdots & \vdots & \ddots & 0 \\
0 & 0 & \cdots & P_{k}\left(x_{N}\right)
\end{array}\right], \mathbf{G}=\left[\begin{array}{c}
g\left(x_{0}\right) \\
g\left(x_{1}\right) \\
\vdots \\
g\left(x_{N}\right)
\end{array}\right], \mathbf{X}=\left[\begin{array}{c}
\mathbf{X}\left(x_{0}\right) \\
\mathbf{X}\left(x_{1}\right) \\
\vdots \\
\mathbf{X}\left(x_{N}\right)
\end{array}\right]=\left[\begin{array}{cccc}
1 & x_{0} & \cdots & x_{0}^{N} \\
1 & x_{1} & \cdots & x_{1}^{N} \\
\vdots & \vdots & \ddots & \vdots \\
1 & x_{N} & \cdots & x_{N}^{N}
\end{array}\right] .
$$

Briefly, Eq.(16) can be written in the form

$$
\mathbf{W A}=\mathbf{G} \text { or }[\mathbf{W} ; \mathbf{G}] ; \mathbf{W}=\sum_{k=0}^{m} \mathbf{P}_{k} \mathbf{X B}\left(\tau_{k}\right) \mathbf{B}^{k} \mathbf{F}^{T}-\sum_{s=0}^{m} \mathbf{X} \mathbf{F}^{T} \mathbf{K}_{L}^{s} \mathbf{Q}_{s} .
$$

Here, Eq.(17) corresponds to a system of $(N+1)$ linear algebraic equations with the unknown Müntz-Legendre coefficients $a_{0}, a_{1}, \ldots, a_{N}$.

By using the relation (8), the matrix form of the conditions (2) becomes

$$
\mathbf{U}_{i} \mathbf{A}=\left[\lambda_{i}\right] \text { or }\left[\mathbf{U}_{i}, \lambda_{i}\right], i=0,1, \ldots, m-1
$$

where

$$
\mathbf{U}_{i}=\left[\begin{array}{llll}
u_{i 0} & u_{i 1} & \ldots & u_{i N}
\end{array}\right]=\left[\sum_{k=0}^{m-1}\left(a_{i k} \mathbf{X}(0)+b_{i k} \mathbf{X}(1)\right)\right] \mathbf{B}^{k} \mathbf{F}^{T}=\left[\lambda_{i}\right], i=0,1, \ldots, m-1 .
$$

To obtain the solution of Eq. (1) under the conditions (2), by replacing the last $m$ rows of matrix (17) by the $m$ row matrices (18) we have the new augmented matrix

$$
\tilde{\mathbf{W}} \mathbf{A}=\tilde{\mathbf{G}} \text { or }[\tilde{\mathbf{W}} ; \tilde{\mathbf{G}}] \text {. }
$$

If $\operatorname{rank} \tilde{\mathbf{W}}=\operatorname{rank}[\tilde{\mathbf{W}} ; \tilde{\mathbf{G}}]=N+1$, the unknown coefficients matrix $\mathbf{A}$ becomes

$$
\mathbf{A}=(\tilde{\mathbf{W}})^{-1} \tilde{\mathbf{G}} \text {. }
$$

Thus, the Müntz-Legendre coefficients matrix $\mathbf{A}$ is uniquely determined. Finally, by substituting the determined coefficients $a_{0}, a_{1}, \ldots, a_{N}$ into Eq.(3), we get the MüntzLegendre polynomial solution

$$
y_{N}(x)=\sum_{n=0}^{N} a_{n} L_{n}(x) .
$$

\section{ERROR ESTIMATION AND IMPROVED APPROXIMATE SOLUTIONS}

In this section, we develop an error estimation for the Müntz-Legendre approximate solution for the problem by means of the residual correction method [10,11] and we improve the approximate solution (19) by using this error estimation. The residual error estimation was presented for the Bessel approximate solutions of the system of the linear multi-pantograph equations [12]. For the problem (1)-(2), we modify the error estimation considered in [10-12].

Let us call $e_{N}(x)=y(x)-y_{N}(x)$ as the error function of the Müntz-Legendre approximation $y_{N}(x)$ to $y(x)$, where $y(x)$ is the exact solution of problem (1)-(2). Hence, $y_{N}(x)$ satisfies the following problem:

$$
\sum_{k=0}^{m} P_{k}(x) y_{N}^{(k)}\left(x+\tau_{k}\right)-\int_{0}^{1} \sum_{s=0}^{m} K_{s}(x, t) y_{N}^{(s)}\left(x+\gamma_{s}\right) d t=g(x)+R_{N}(x) \quad 0 \leq x, t \leq 1
$$




$$
\sum_{k=0}^{m-1}\left(a_{j k} y_{N}^{(k)}(0)+b_{j k} y_{N}^{(k)}(1)\right)=\lambda_{j}, j=0,1, \ldots, m-1 .
$$

can be obtained by substituting $y_{N}(x)$ into the Eq. (1) and in here $R_{N}(x)$ is the residual function associated with $y_{N}(x)$.

By using the method defined in Section 3, we purpose to find an approximation $e_{N, M}(x)$ to the $e_{N}(x)$.

Subtracting (20) and (21) from (1) and (2), respectively, the error function $e_{N}(x)$ satisfy the equation

$$
\sum_{k=0}^{m} P_{k}(x) e_{N}^{(k)}\left(x+\tau_{k}\right)-\int_{0}^{1} \sum_{s=0}^{m} K_{s}(x, t) e_{N}^{(s)}\left(x+\gamma_{s}\right) d t=-R_{N}(x) \quad 0 \leq x, t \leq 1
$$

with the homogeneous conditions

$$
\sum_{k=0}^{m-1}\left(a_{j k} e_{N}^{(k)}(0)+b_{j k} e_{N}^{(k)}(1)\right)=0, j=0,1, \ldots, m-1 .
$$

Solving the error problem (22)-(23) by our method, we obtain the approximation $e_{N, M}(x)$ to $e_{N}(x)$.

Consequently, we have the improved approximate solution

$$
y_{N, M}(x)=y_{N}(x)+e_{N, M}(x) \text {. }
$$

Note that if the exact solution of the problem is not known, then we can estimate the error function by $e_{N, M}(x)$.

\section{NUMERICAL APPLICATION}

In this section, we consider a problem to demonstrate the effectiveness of the method, the error estimation and the residual correction.

Example. Now, let us solve linear delay Fredholm integro-differential equation

$$
y^{(4)}(x)-y^{(2)}(x-1)+x y(x+0.5)=g(x)+\int_{0}^{1} x \cos (t) y^{(1)}(t+1) d t+\int_{0}^{1} x \sin (t) y^{(2)}(t-0.5) d t, \quad 0 \leq x, t \leq 1
$$

under the initial conditions $y(0)=0, y^{(1)}(0)=1, y^{(2)}(0)=0, y^{(3)}(0)=-1$. Here, $g(x)=\sin (x)+\sin (x-1)+x \sin (x+1 / 2)+\frac{1}{4} x \sin (1)-\frac{1}{2} x \cos (1)-\frac{1}{4} x \sin (3)-\frac{1}{4} x \sin (1 / 2)$ $+\frac{1}{2} x \cos (1 / 2)-\frac{1}{4} x \sin (3 / 2)$. For this problem, $m=4, \tau_{0}=0.5, \tau_{1}=-1, \tau_{4}=0, P_{0}=x$, $P_{1}=-1, \quad P_{4}=1, \quad \gamma_{1}=1, \gamma_{2}=-0.5, \quad K(x, t)=x \cos (t)$ and $K_{2}(x, t)=x \sin (t)$.

We note that the exact solution of the problem is $y(x)=\sin (x)$. 
Table 1. Numerical results of the error functions for $N=5,8$ and $M=7,9$ of Eq. (24)

\begin{tabular}{lllllll}
\hline & \multicolumn{2}{l}{ Actual absolute errors } & \multicolumn{2}{l}{ Estimated absolute errors } & \multicolumn{2}{l}{ Corrected absolute errors } \\
\cline { 2 - 7 }$x_{i}$ & $\left|e_{5}\left(x_{i}\right)\right|$ & $\left|e_{8}\left(x_{i}\right)\right|$ & $\left|e_{5,7}\left(x_{i}\right)\right|$ & $\left|e_{8,9}\left(x_{i}\right)\right|$ & $\left|E_{5,7}\left(x_{i}\right)\right|$ & $\left|E_{8,9}\left(x_{i}\right)\right|$ \\
\hline 0 & $1.2406 \mathrm{e}-015$ & $2.2329 \mathrm{e}-015$ & $1.7745 \mathrm{e}-019$ & $6.2660 \mathrm{e}-019$ & $1.2408 \mathrm{e}-015$ & $2.2323 \mathrm{e}-015$ \\
0.2 & $1.2020 \mathrm{e}-005$ & $9.2512 \mathrm{e}-007$ & $1.2815 \mathrm{e}-005$ & $1.3853 \mathrm{e}-006$ & $7.9556 \mathrm{e}-007$ & $4.6017 \mathrm{e}-007$ \\
0.4 & $1.6817 \mathrm{e}-004$ & $1.9010 \mathrm{e}-005$ & $1.8080 \mathrm{e}-004$ & $2.4918 \mathrm{e}-005$ & $1.2634 \mathrm{e}-005$ & $5.9080 \mathrm{e}-006$ \\
0.6 & $7.2667 \mathrm{e}-004$ & $1.1940 \mathrm{e}-004$ & $7.9102 \mathrm{e}-004$ & $1.4216 \mathrm{e}-004$ & $6.4355 \mathrm{e}-005$ & $2.2757 \mathrm{e}-005$ \\
0.8 & $1.8910 \mathrm{e}-003$ & $4.5459 \mathrm{e}-004$ & $2.0979 \mathrm{e}-003$ & $5.0473 \mathrm{e}-004$ & $2.0684 \mathrm{e}-004$ & $5.0138 \mathrm{e}-005$ \\
1 & $3.5897 \mathrm{e}-003$ & $1.3046 \mathrm{e}-003$ & $4.1067 \mathrm{e}-003$ & $1.3761 \mathrm{e}-003$ & $5.1705 \mathrm{e}-004$ & $7.1436 \mathrm{e}-005$ \\
\hline
\end{tabular}

For $(N, M)=(5,7)$ and $(N, M)=(8,9)$, we solve the above problem. In Table 1 , we give the absolute errors (actual, estimation and correction) of this problem.

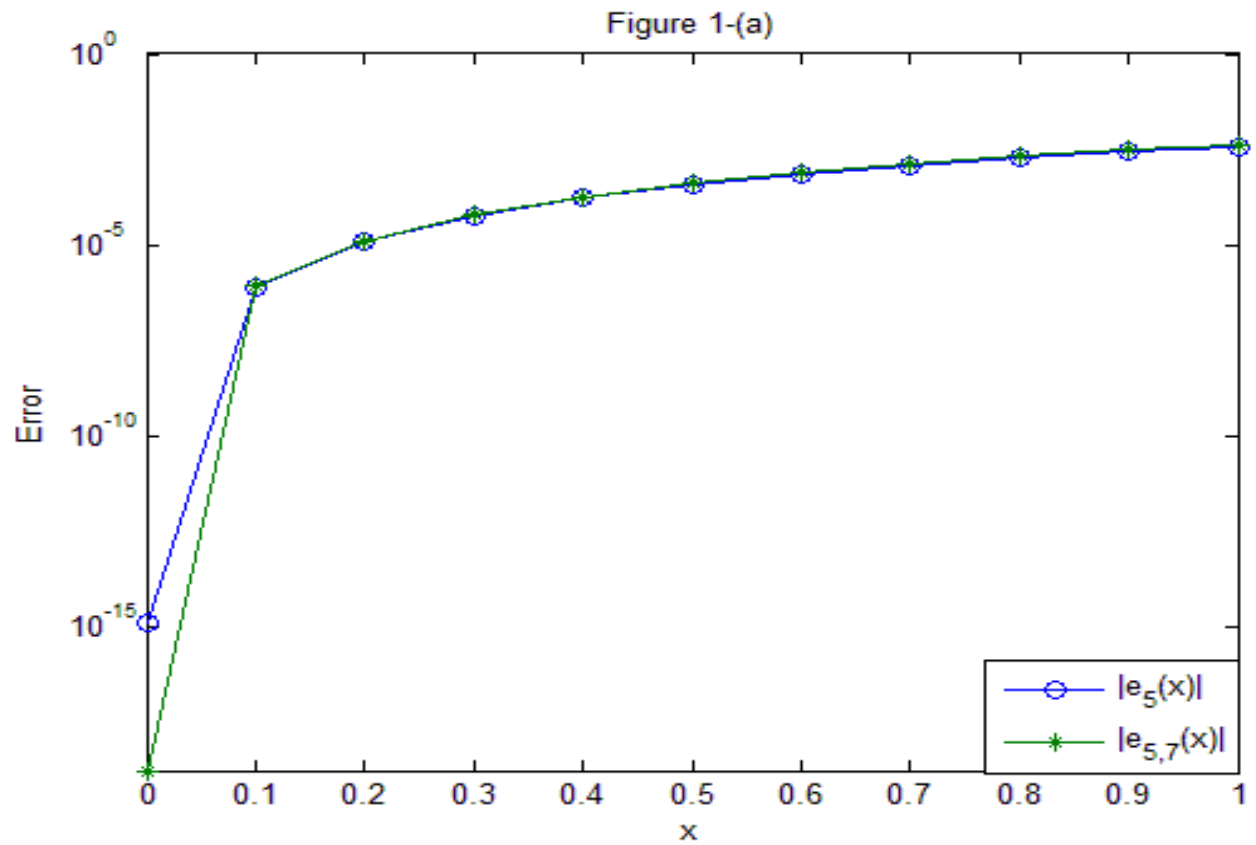

Figure 1-(a). Comparison of the actual absolute error function $\left|e_{5}(x)\right|$ and the estimated absolute error function $\left|e_{5,7}(x)\right|$ for $(N, M)=(5,7)$. 


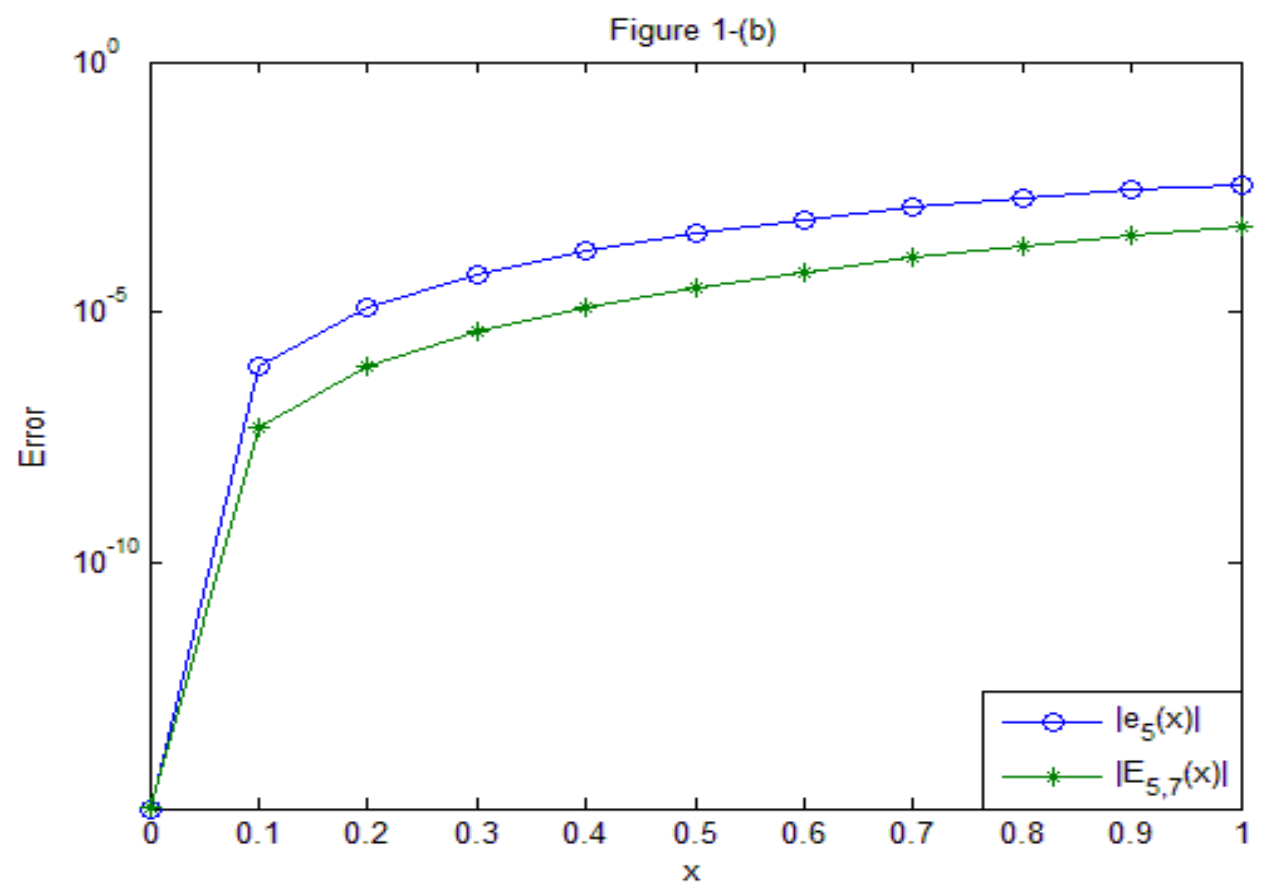

Figure 1-(b). Comparison of the actual absolute error function $\left|e_{5}(x)\right|$ of the approximate solution $y_{5}(x)$ and the actual absolute error function $\left|E_{5,7}(x)\right|$ of the improved approximate solution $y_{5,7}(x)$ for $(N, M)=(5,7)$.

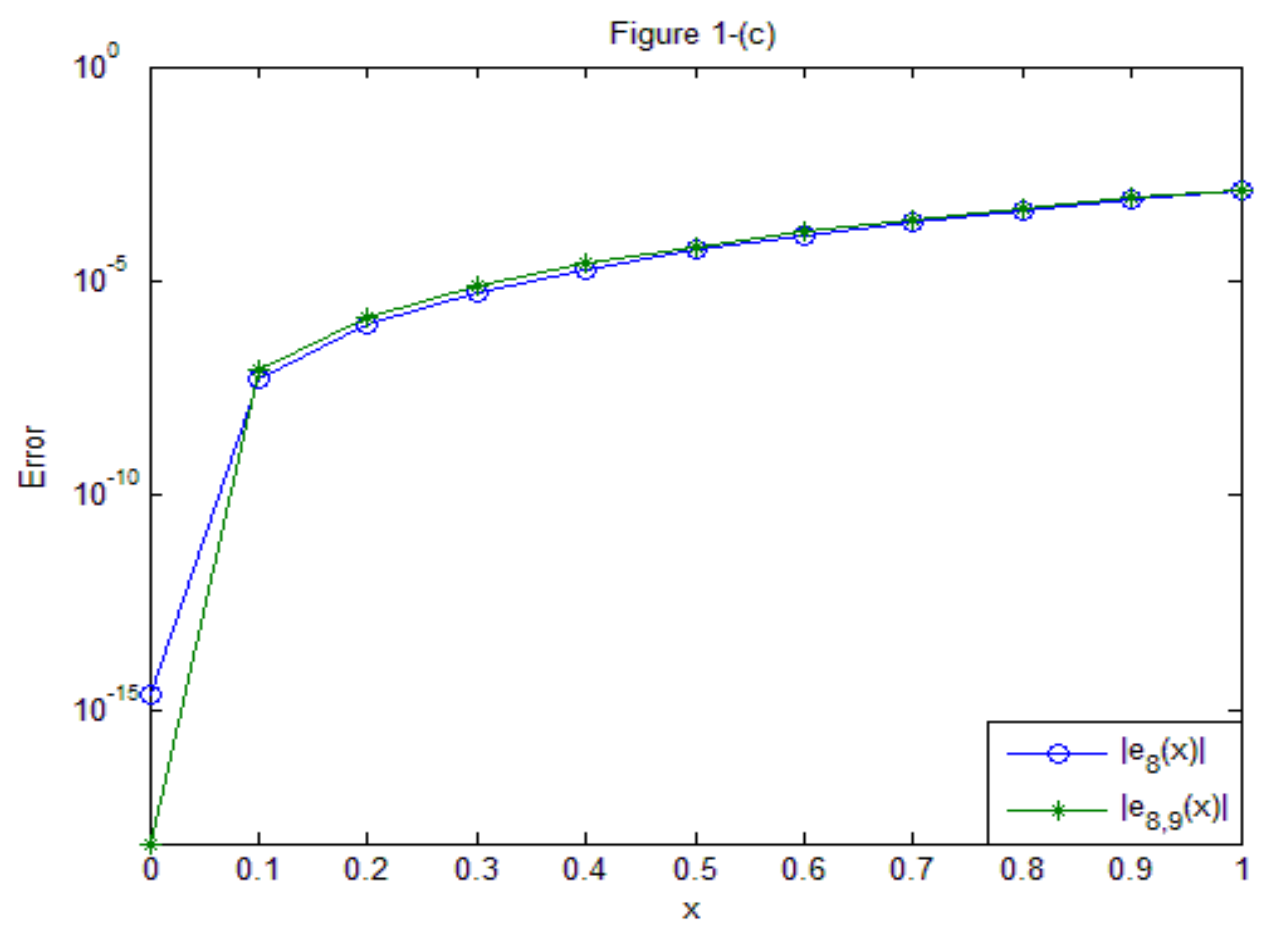

Figure 1-(c). Comparison of the actual absolute error function $\left|e_{8}(x)\right|$ and the estimated absolute error function $\left|e_{8,9}(x)\right|$ for $(N, M)=(8,9)$. 


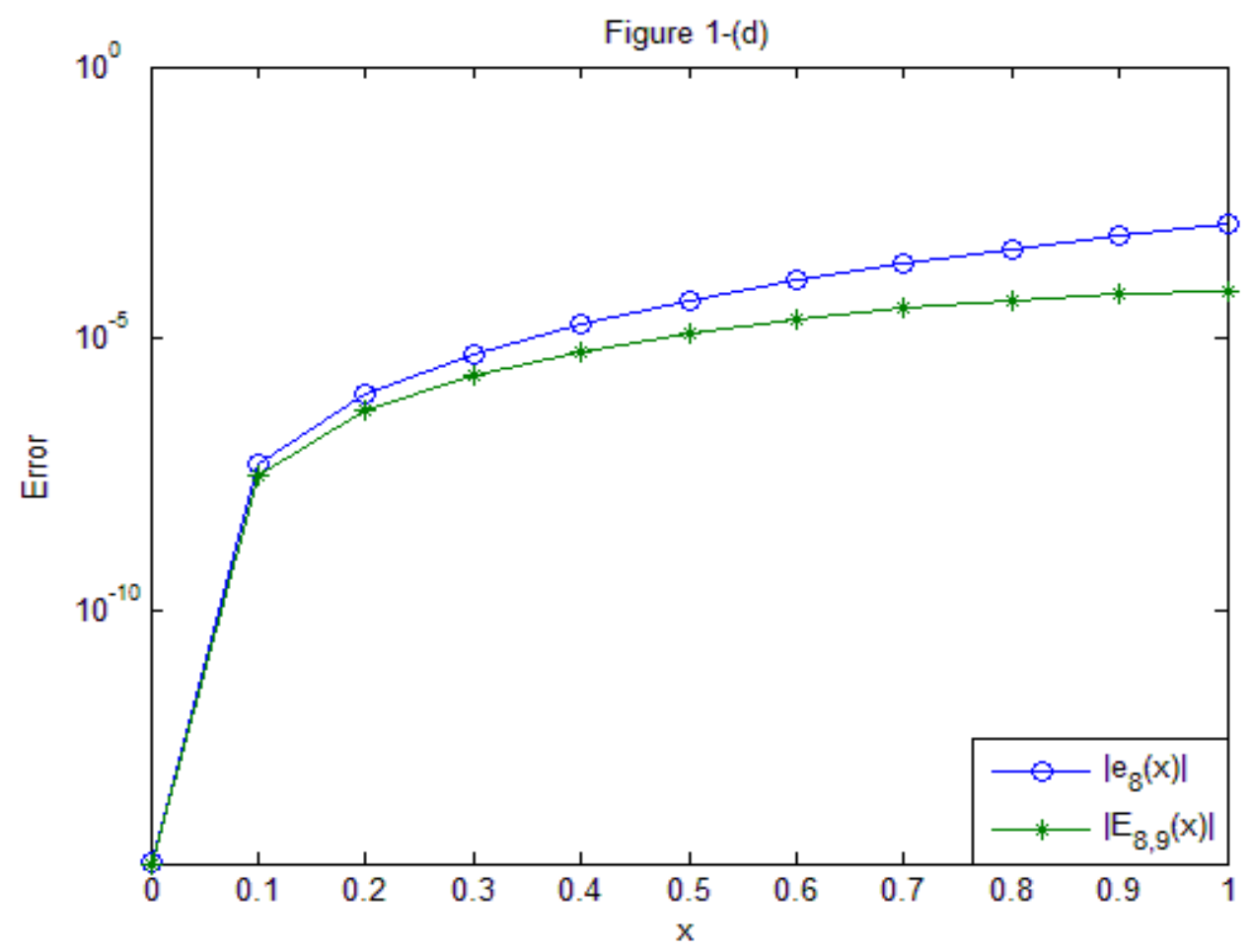

Figure 1-(d). Comparison of the actual absolute error function $\left|e_{8}(x)\right|$ of the approximate solution $y_{8}(x)$ and the actual absolute error function $\left|E_{8,9}(x)\right|$ of the improved approximate solution $y_{8,9}(x)$ for $(N, M)=(8,9)$.

\section{CONCLUSIONS}

In this paper, we presented the Müntz-Legendre collocation scheme of the linear delay Fredholm integro-differential equations. In addition, we constructed an error problem by means of the residual error function and this problem is solved by Müntz-Legendre collocation scheme. Hence, the Müntz-Legendre polynomial solution of this error problem is an approximation for the actual error function. Finally, by summing the estimated error function and Müntz-Legendre polynomial solution, the corrected approximate solution is obtained. We gave the application of our works for an example. In this application, we observed from example that actual and estimated errors are very close and the improvement is quite effective. Therefore, we note that the error estimation can be used for measurement of the reliability of the considered problem, when an exact solution of any problem is not available.

Acknowledgments-The first author is supported by the Scientific Research Project Administration of Akdeniz University. 


\section{REFERENCES}

1. S. Yalçınbaş, H. H. Sorkun and M. Sezer, Legendre polynomial solutions of highorder linear Fredholm integro-differential equations, Applied Mathematics and Computation 210, 334-349, 2009.

2. M. Sezer and A. Akyüz-Daşcioğlu, A Taylor method for numerical solution of generalized pantograph equations with lineer functional argument, Journal of Computational and Applied Mathematics 200, 217-225, 2007.

3. S. Yalçınbaş, N. Özsoy and M. Sezer, Approximate solution of higher order linear differential equations by means of a new rational chebyshev collocation method, Mathematical and Computational Applications 15, 45-56, 2010.

4. Ş. Yüzbaşı, N. Şahin and M. Sezer, A Bessel collocation method for numerical solution of generalized pantograph equations, Numerical Methods for Partial Differential Equations 28, 1105-1123, 2012.

5. M. Gülsu, B. Gürbüz, Y. Öztürk and M. Sezer, Laguerre polynomial approach for solving linear delay difference equations, Applied Mathmematics and Computation 217, 6765-6776, 2011.

6. Ú. Stefansson, Asymptotic properties of Müntz orthogonal polynomials, $\mathrm{PhD}$. Thesis, Georgia Institute of Technology, Atlanta, 124 pages, 2010.

7. Ş. Yüzbaşı, N. Şahin and M. Sezer, Numerical solutions of systems of linear Fredholm integro-differential equations with Bessel polynomials bases, Computers and Mathematics with Applications 61, 3079-3096, 2011.

8. S. Yalçınbaş, M. Sezer, The approximate solutions of higher-order linear VolterraFredholm integro-differential equations in terms of Taylor polynomials, Applied Mathematics and Computation 112, 291-308, 2000.

9. M. Gülsu, M. Sezer, The approximate solution of high-order linear difference equations with variable coefficients in terms of Taylor polynomials, Applied Mathematics and Computation 168, 76-88, 2005.

10. F. A. Oliveira, Collacation and residual correction, Numerische Mathematik 36, 2731,1980 .

11. İ. Çelik, Collocation method and residual correction using Chebyshev series, Applied Mathmematics and Computation 174, 910-920, 2006.

12. Ş. Yüzbaşı, An efficient algorithm for solving multi-pantograph equation systems, Computers and Mathematics with Applications 64, 589-603, 2012. 